$$
C=\left(\begin{array}{ll}
L & M \\
N & P
\end{array}\right)
$$

$L, M$, and so on, being in $\Sigma_{n}$, it follows that $M^{\prime}=-M, N^{\prime}=-N$, $P=L^{\prime}$ and conversely any matrix of this form satisfies the condition. If we specialize the indeterminates so that $M=N=0$, we obtain a matrix with irreducible minimum polynomial of degree $n$. It follows that $\psi(x)$ for the general matrix has degree not less than $n$ and hence $\psi(x)=\phi(x)$ and $f(x)=[\phi(x)]^{2}$.

THEOREM. If $B$ is a matrix of $2 n$ rows and columns with elements in a field of characteristic not equal to 2 such that $R^{-1} B^{\prime} R=B$, where $R$ is any non-singular skew symmetric matrix, then the characteristic polynomial $f(x)$ of $B$ has the form $[\phi(x)]^{2}$, where the coefficients of $\phi(x)$ are polynomials in the elements of $B$ and $\phi(B)=0$. If the elements of the general matrix $B$ are regarded as indeterminates, then $\phi(x)$ is irreducible.

University of North Carolina

\title{
THE EULER-MACLAURIN SUMMATION FORMULA*
}

\section{TOMLINSON FORT}

In this paper an extension of the classical Euler-Maclaurin summation formula $\dagger$ is made to multiple sums. Bernoulli polynomials and numbers of higher order as defined by Nörlund $\ddagger$ enter into the formula and Bernoulli numbers of negative order enter into the proof. Nörlund obtains $\S$ a formula for $\phi(x+\omega)$ in terms of Bernoulli numbers of higher order, and this is called by him an extension of the Euler-Maclaurin formula. His formula permits the ready building up of a simple sum. This is not true, however, of a multiple sum. Steffensen $\|$ calls attention to the fact that a multiple sum can be reduced by summation by parts to a simple sum and the EulerMaclaurin formula for the simple sum used. However, the function to be summed is changed by his suggested transformation and he develops no general formula, nor does he suggest the use of Bernoulli numbers of higher order.

The formula developed in the present paper is equally as easy of

* Presented to the Society, October 29, 1938.

† See, for example, D. Seliwanoff, Lehrbuch der Differenzenrechnung, p. 48, where it is called the Euler sum formula. It is sometimes also called the Maclaurin sum formula. See W. B. Ford, Studies on Divergent Series and Summability.

$\ddagger$ N. E. Nörlund, Differenzenrechnung, p. 129.

\$ Loc. cit., p. 160.

\| J. F. Steffensen, Interpolation, p. 136. 
application as the classical formula. It is applied to one example. Throughout the paper use is freely made of the properties which Nörlund has developed of $B_{n}{ }^{(l)}(x)$ and $B_{n}{ }^{(l)}$, the Bernoulli polynomials and numbers of order $l$ and degree $n$.

1. The formula. Let

$$
\begin{aligned}
f(x)= & P_{0}(n+k)(n+k-1) \cdots n x^{n-1} \\
& +P_{1}(n+k-1) \cdots(n-1) x^{n-2}+\cdots \\
& +P_{n-1}(k+1) \cdots 2 \cdot 1
\end{aligned}
$$

where the $P$ 's are constants, and let $\omega$ be a difference interval. Then*

$$
\begin{aligned}
& \Phi_{1}(f(x)) \equiv \omega \sum_{x_{1}=0}^{x-\omega} f\left(x_{1}\right)=P_{0}(n+k)(n+k-1) \cdots(n+1)\left[B_{n}^{(1)}(x)\right. \\
& \left.-B_{n}^{(1)}\right]+\cdots+P_{n-1}(k+1) \cdots 2\left[B_{1}^{(1)}(x)-B_{1}^{(1)}\right] \text {, } \\
& \Phi_{2}(f(x)) \equiv \omega^{2} \sum_{x_{1}=0}^{x-\omega} \sum_{x_{2}=0}^{x_{1}-\omega} f\left(x_{2}\right) \\
& =P_{0}(n+k) \cdots(n+2)\left[B_{n+1}^{(2)}(x)-B_{n+1}^{(2)}\right]+\cdots \\
& +P_{n-1}(k+1) \cdots 3\left[B_{2}^{(2)}(x)-B_{2}^{(2)}\right]-\left[P_{0}(n+k)\right. \\
& \left.\cdots(n+1) B_{n}^{(1)}+\cdots+P_{n-1}(k+1) \cdots 2 B_{1}^{(1)}\right] x \text {, } \\
& \Phi_{k+1}(f(x)) \equiv \omega^{k+1} \sum_{x_{1}=0}^{x-\omega} \cdots \sum_{x_{k+1}=0}^{x_{k}-\omega} f\left(x_{k+1}\right)=P_{0}\left[B_{n+k}^{(k+1)}(x)-B_{n+k}^{(k+1)}\right]+\cdots \\
& +P_{n-1}\left[B_{k+1}^{(k+1)}(x)-B_{k+1}^{(k+1)}\right] \\
& -\left[P_{0}(n+k) B_{n+k-1}^{(k)}+\cdots+P_{n-1}(k+1) B_{k}^{(k)}\right] x \\
& \text { - }\left[P_{0}(n+k)(n+k-1) B_{n+k-2}^{(k-1)}+\cdots\right. \\
& \left.+P_{n-1}(k+1) k B_{k-1}^{(k-1)}\right] \frac{x(x-\omega)}{2 !}-\cdots \\
& -\left[P_{0}(n+k) \cdots(n+1) B_{n}^{(1)}+\cdots\right. \\
& \left.+P_{n-1}(k+1) \cdots 2 B_{1}^{(1)}\right] \cdot \frac{x(x-\omega) \cdots(x-(k-2) \omega)}{(k-1) !} \text {. }
\end{aligned}
$$

* Notation throughout the paper is $\sum_{x-a}^{x-\omega} f(x)=f(a)+f(a+\omega)+\cdots+f(x-\omega)$. We also use the convention $\sum_{x_{1}=a}^{x-\omega} \sum_{x_{2}=a}^{x_{1}-\omega} f\left(x_{2}\right)=0$ if $x-\omega \leqq a$. It is well to remark that in case $f(x) \equiv f\left(x_{1}\right)$ is a polynomial and $\sum_{x_{1}=0}^{x-\omega} f_{n-1}\left(x_{1}\right)=f_{n}(x)$, then $f_{n}(0)=f_{n}(\omega)=\cdots$ $=f_{n}((n-1) \omega)=0$. Consequently, $\sum_{x_{1}=0}^{x-x_{1}=0} \cdots \sum_{x_{n}=0}^{x_{n-1}-\omega} f\left(x_{n}\right)$ can be evaluated by successive summations without calling into play any convention. 
Rearrange the terms in (2) and we have

$$
\begin{aligned}
\Phi_{k+1}(f(x))= & B_{0}^{(k+1)}\left[P_{0} x^{n+k}+P_{1} x^{n+k-1}+\cdots+P_{n-1} x^{k+1}\right] \\
& +B_{1}^{(k+1)}\left[P_{0}(n+k) x^{n+k-1}+\cdots+P_{n-1}(k+1) x^{k}\right] \\
& +B_{2}^{(k+1)}\left[P_{0} \frac{(n+k)(n+k-1)}{2 !} x^{n+k-2}+\cdots\right. \\
& \left.+P_{n-1} \frac{(k+1) k}{2 !} x^{k-1}\right]+\cdots+B_{n+k}^{(k+1)}\left[P_{0}\right] \\
& -\left[P_{n-1} B_{k+1}^{(k+1)}+\cdots+P_{0} B_{n+k}^{(k+1)}\right] \\
& -\left[P_{n-1}(k+1) B_{k}^{(k)}+\cdots+P_{0}(n+k) B_{n+k-1}^{(k)}\right] x-\cdots \\
& -\left[P_{n-1}(k+1) \cdots 2 B_{1}^{(1)}+\cdots\right. \\
+ & \left.P_{0}(n+k) \cdots(n+1) B_{n}^{(1)}\right] \\
& \cdot \frac{x(x-\omega) \cdots(x-(k-1) \omega)}{k !}
\end{aligned}
$$

From this, by inspection,

$$
\begin{aligned}
\Phi_{k+1}(f(x))= & B_{0}^{(k+1)} \int_{0}^{x} \int_{0}^{x_{1}} \cdots \int_{0}^{x_{k}} f\left(x_{k+\mathrm{i}}\right) d x_{1} d x_{2} \cdots d x_{k+1} \\
& +B_{1}^{(k+1)} \int_{0}^{x} \cdots \int_{0}^{x_{k-1}} f\left(x_{k}\right) d x_{1} \cdots d x_{k} \\
& +\frac{1}{2 !} B_{2}^{(k+1)} \int_{0}^{x} \cdots \int_{0}^{x_{k-2}} f\left(x_{k-1}\right) d x_{1} \cdots d x_{k-1}+\cdots \\
& +\frac{1}{k !} B_{k}^{(k+1)} \int_{0}^{x} f\left(x_{1}\right) d x_{1}+\frac{1}{(k+1) !} B_{k+1}^{(k+1)} f(x)+\cdots \\
& +\frac{1}{(n+k) !} B_{n+k}^{(k+1)} f^{(n-1)}(x) \\
& -\left[B_{k+1}^{(k+1)} \frac{f(0)}{(k+1) !}+\cdots+B_{n+k}^{(k+1)} \frac{f^{(n-1)}(0)}{(n+k) !}\right] \\
& -\left[B_{k}^{(k)} \frac{f(0)}{k !}+\cdots+B_{n+k-1}^{(k)} \frac{f^{(n-1)}(0)}{(n+k-1) !}\right] x-\cdots \\
& -\left[B_{1}^{(1)} \frac{f(0)}{1 !}+\cdots+B_{n}^{(1)} \frac{f^{(n-1)}(0)}{n !}\right] \\
& \cdot \frac{x(x-\omega) \cdots(x-(k-1) \omega)}{k !} \cdot
\end{aligned}
$$


This is the desired generalization of the Euler-Maclaurin formula. As developed for (1), it is a polynomial identity.

We now assume $f(x)$ not a polynomial and proceed to an examination of the remainder when the right-hand member of (3) replaces $\Phi_{k+1}(f(x))$. We assume $x, \omega$ and $f(x)$ real and $x \geqq(k+1) \omega$, also that all derivatives, which enter, exist. Denote the right-hand member of (3) by $\Psi_{k+1}$. Let $R_{n}(x)=\Phi_{k+1}-\Psi_{k+1}$. We now use a generalization* of Taylor's formula and obtain

$$
\begin{aligned}
-\Delta^{k+1} R_{n}(x) & =-\omega^{k+1} f(x)+B_{0}^{k+1}\left[f(x)+\frac{\Delta^{k+1} 0^{k+2}}{(k+2) !} f^{\prime}(x)\right. \\
& +\frac{\Delta^{k+1} 0^{k+3}}{(k+3) !} f^{\prime \prime}(x)+\cdots \\
& \left.+\frac{\Delta^{k+1} 0^{n+k}}{(n+k) !} f^{(n-1)}(x)+R_{0}^{(k+1)}\right] \\
& +B_{1}^{(k+1)}\left[f^{\prime}(x)+\frac{\Delta^{k+1} 0^{k+2}}{(k+2) !} f^{\prime \prime}(x)+\cdots\right. \\
& \left.+() f^{(n-1)}(x)+R_{1}^{(k+1)}\right] \\
& +\frac{1}{2 !} B_{2}^{(k+1)}\left[f^{\prime \prime}(x)+\cdots+() f^{(n-1)}(x)+R_{2}^{(k+1)}\right] \\
& +\cdots+\frac{1}{(n+k) !} B_{n+k}^{(k+1)}\left[R_{n+k}^{(k+1)}\right] .
\end{aligned}
$$

Here $†$

$$
\begin{aligned}
R_{j}^{(k+1)}= & \frac{1}{(n+k-j) !} \sum_{i=0}^{k}(-1)^{i} \\
& \cdot \int_{0}^{(k+1-i) \omega} C_{k+1, k+1-i} t^{n+k-j} f^{(n)}(x+(k+1-i) \omega-t) d t .
\end{aligned}
$$

We note that $B_{0}^{(i)}=1, i=1,2, \cdots$. We also now note the follow-

* George Boole, Calculus of Finite Differences, p. 24. In the present paper in calculating $\Delta^{n} 0^{n}$ the difference interval is $\omega$.

$\dagger$ This is readily obtained if we write

$$
\Delta^{k+1} Q(x)=\sum_{i=0}^{k}(-1)^{i} C_{k+1, k+1-i}[Q(x+(k+1-i) \omega)-Q(x)]
$$

and use the integral form for the remainder when $Q(x+(k+1-i) \omega)-Q(x)$ is developed by Taylor's formula. Here, as throughout the paper, $C_{n, m}$ denotes a binomial coefficient. 
ing relation between the numbers* $\Delta^{n} 0^{n}$ and the Bernoulli numbers of negative order as defined by Nörlund with all difference intervals equal:

$$
\omega^{n} B_{\nu}^{(-n)}=\frac{\nu !}{(\nu+n) !} \Delta^{n} 0^{\nu+n} .
$$

Substituting in (4) and rearranging terms we have:

$$
\begin{aligned}
-\Delta^{k+1} R_{n}(x)=\omega^{k+1} \sum_{j=1}^{n} \frac{f^{i}(x)}{j !} \sum_{i=0}^{j} & C_{j, i} B_{i}^{(k+1)} B_{j-i}^{-(k+1)} \\
& +\sum_{j=0}^{n+k} \frac{1}{j !} B_{j}^{(k+1)} R_{j}^{(k+1)} .
\end{aligned}
$$

But the first sum in the right-hand member is zero. $\dagger$ Hence we obtain

$$
\Delta^{k+1} R_{n}(x)=-\sum_{j=0}^{n+k} \frac{1}{j !} B_{j}^{(k+1)} R_{j}^{(k+1)} .
$$

Substituting for $R_{j}^{(k+1)}$ its value from (5), we have

$$
\Delta^{k+1} R_{n}(x)=\frac{-1}{(n+k) !} \sum_{i=0}^{k} \int_{0}^{(k+1-i) \omega}(-1)^{i} C_{k+1, k+1-i} B_{n+k}^{(k+1)}(t)
$$

$$
f^{(n)}(x+(k+1-i) \omega-t) d t
$$

Moreover,

$$
R_{n}(0)=\Delta R_{n}(0)=\cdots=\Delta^{k} R_{n}(0)=0 .
$$

To show this, refer to the definition of $R_{n}$ as $\Phi_{k+1}-\Psi_{k+1}$ and use (3). It is immediate that $R_{n}(0)=0$. To show that $\Delta R_{n}(0), \cdots, \Delta^{k} R_{n}(0)$ are also zero, let $f(x)$ be a polynomial of arbitrary degree. Develop $\Delta^{i} R_{n}(x), i=1, \cdots, k$, by the extended Taylor's series as given by Boole. $\ddagger$ All series are finite. Let $x=0$. We have a polynomial identity in $f(0), f^{\prime}(0), \cdots$. Since $f(0), f^{\prime}(0), \cdots$ are arbitrary, their coefficients must be zero. These are expressions in the Bernoulli numbers that are independent of the function considered. Hence,

$$
R_{n}(x)=\sum_{x_{1}=0}^{x-\omega} \sum_{x_{2}=0}^{x_{1}-\omega} \cdots \sum_{x_{k+1}=0}^{x_{k}-\omega} \Delta^{k+1} R_{n}\left(x_{k+1}\right),
$$

* Boole, loc. cit., p. 28.

$\dagger$ Nörlund, loc. cit., p. 141.

$\ddagger$ Loc. cit., p. 24 
where $\Delta^{k+1} R_{n}(x)$ is given by (6). This form for $R_{n}(x)$ reduces to the classical Jacobi form* in case $k=0$.

The transformation of the interval $(0, x)$ into $(a, y)$ in $(3)$ and $(7)$ by means of the transformation $y=a+x$ is immediate.

2. An example. Consider $\Phi_{k+1}\left(1 /(x+1)^{\alpha}\right)$, with $\omega=1, \alpha>0$. Then

$$
R_{n}(x)=-\frac{1}{(n+k) !} \sum_{i=0}^{k} \int_{0}^{k+1-i} \sum_{x_{1}=0}^{x-1} \cdots \sum_{x_{k+1}=0}^{x_{k}-1} L(x, t, i, n) d t
$$

where

$$
\begin{aligned}
L \equiv(-1){ }^{i+n} C_{k+1, k+1-i} B_{n+k}^{(k+1)}(t) \alpha(\alpha+1) \\
\cdots(\alpha+n-1)(x+k+2-i-t)^{-(\alpha+n)}
\end{aligned}
$$

Adopting the notation $x^{(l)}=x(x-1) \cdots(x-l+1)$, it is readily shown by mathematical induction on $k$ that

$$
\sum_{x_{1}=0}^{x-1} \cdots \sum_{x_{k+1}=0}^{x_{k}-1} L=A_{0} x^{(k)}+A_{1} x^{(k-1)}+\cdots+A_{k}+M
$$

where the $A$ 's are independent of $x$ and

From this

$$
M=(-1)^{k+1} \sum_{x_{1}=x}^{\infty} \cdots \sum_{x_{k+1}=x_{k}}^{\infty} L .
$$

$$
|M| \leqq \int_{x-1}^{\infty} \cdots \int_{x_{k}-1}^{\infty} \frac{L^{\prime}}{\left(x_{k+1}+1\right)^{\alpha+n}} d x_{1} \cdots d x_{k+1} \leqq \frac{L^{\prime \prime}}{(x+1)^{\alpha+n-k-1}},
$$

where $L^{\prime}$ and $L^{\prime \prime}$ are certain positive constants. Here, in order to assure the convergence of all series under discussion, it is necessary to assume $n>k+1-\alpha$. We next integrate (10) as indicated in (8), and let

$$
M^{\prime}=\int_{0}^{k+1-i} M d t
$$

Then $\left|M^{\prime}\right| \leqq(k+1-i) L^{\prime \prime} /(x+1)^{\alpha+n-k-1}$. Let

$$
M^{\prime \prime \prime}=\frac{-1}{(n+k) !} \sum_{i=0}^{k} M^{\prime}
$$

Then, $\left|M^{\prime \prime}\right| \leqq L^{\prime \prime \prime} /(x+1)^{\alpha+n-k-1}$, where $L^{\prime \prime \prime}$ is a constant. Let

* Seliwanoff, loc. cit., p. 50, formula (4). 


$$
\begin{aligned}
& D_{j}=\frac{-1}{(n+k) !} \sum_{i=0}^{k} \int_{0}^{k+1-i} A_{j} d t \\
& C_{j}=D_{i}-\frac{1}{(k-j) !}\left[B_{j+1}^{(j+1)} \frac{f(0)}{(j+1) !}+\cdots+B_{n+j}^{(j+1)} \frac{f^{(n-1)}(0)}{(n+j) !}\right] .
\end{aligned}
$$

Then

$$
\begin{aligned}
\Phi_{k+1}\left(\frac{1}{(x+1)^{\alpha}}\right)= & C_{0} x^{(k)}+C_{1} x^{(k-1)}+\cdots+C_{k} \\
& +B_{0}^{(k+1)} \int_{0}^{x} \cdots \int_{0}^{x_{k}} \frac{1}{\left(x_{k+1}+1\right)^{\alpha}} d x_{1} \cdots d x_{k+1} \\
& +\cdots+\frac{1}{k !} B_{k}^{(k+1)} \int_{0}^{x} \frac{1}{\left(x_{1}+1\right)^{\alpha}} d x_{1} \\
& +\frac{1}{(k+1) !} B_{k+1}^{(k+1)} \frac{1}{(x+1)^{\alpha}}+\cdots \\
& +\frac{1}{(n+k) !} B_{n+k}^{(k+1)} \frac{\alpha(\alpha+1) \cdots(\alpha+n-2)}{(x+1)^{\alpha+n-1}}+M^{\prime \prime} .
\end{aligned}
$$

We readily show that the $C$ 's are independent of $n$. Equate right-hand members of (11) for different values of $n$. Cancel integral terms. Divide through by $x^{k}$ and allow $x$ to become infinite. Cancel $C_{0} x^{k}$ from both members and repeat. The $C$ 's can be calculated for particular values of $k$ and $\alpha$. In some instances the calculation may be precise, in others necessarily approximate. For example, if $\alpha=1$ and $k=0$, $C_{0}$ is Euler's constant. If $k=1$ and $\alpha=1, C_{0}$ is Euler's constant and $C_{1}=-1 / 2$. Values for $L^{\prime \prime \prime}$ can be calculated for particular values of $n, k$ and $\alpha$, using (9). This process depends upon determination of an upper bound for the Bernoulli polynomial $B_{n+k}^{(k+1)}(t)$, when $0 \leqq t \leqq k+1$. This can be done from the known form of the polynomial and relations connecting the Bernoulli numbers. However, for large values of $n$ the numerical work is excessive.

LEHIGH UNIVERSITY 